\title{
Band-Engineered Structural Design and Characterization of Deep-Ultraviolet Light-Emitting Diodes
}

\author{
Yen-Kuang Kuo ${ }^{1}$ and Jih-Yuan Chang ${ }^{2}$ \\ ${ }^{1}$ Department of Physics / National Changhua University of Education \\ No.1, Jin-De Road, Changhua City, Taiwan \\ ykuo@cc.ncue.edu.tw \\ ${ }^{2}$ Center for Teacher Education / National Changhua University of Education \\ No.1, Jin-De Road, Changhua City, Taiwan \\ jihyuan67@gmail.com
}

\section{Extended Abstract}

In conventional structures of deep-ultraviolet light-emitting diodes (DUV LEDs), the confinement capability could be promoted by heightening the potential barrier of quantum barriers (QBs) or electron-blocking layer (EBL) with the use of high Al composition. However, the resulted high built-in polarization field forms a trade-off effect to the carrier confinement of AlGaN DUV LEDs. In III-nitride DUV LEDs grown along the $c$-axis, massive polarization-induced sheet charges arising from the spontaneous polarization and piezoelectric polarization of lattice-mismatched adjacent layers accumulate in heterojunctions and form built-in polarization field [1]. The polarization field severely deforms the energy band profile that consequently affects the transport, injection and confinement of carriers, which in turn degrades the radiative recombination efficiency [2,3]. There thus exist joint effects, including the variations of potential barrier height and polarization field, when varying the structure of DUV LEDs. In this study, band-engineered structural design for DUV LEDs is performed numerically with the APSYS simulation program. Specifically, the n-AlGaN layers, QBs and EBLs are significant factors for the energy band profile of the active region in DUV LEDs. However, comprehensive studies regarding the joint effects of aforementioned three structural parameters for their impacts on the DUV LED performance are rarely available at present. This work systematically investigates the DUV LED structures with various n-AlGaN layers, QBs and EBLs in order to achieve the optimized LED structures with efficient carrier confinement and superior output performance. Simulation results reveal that the light output power increases with increasing $\mathrm{Al}$ composition of QBs when the $\mathrm{Al}$ composition of EBL is relatively low, while the light output power decreases with increasing $\mathrm{Al}$ composition of QBs when the $\mathrm{Al}$ composition of EBL is relatively high. As for the DUV LEDs with an EBL of medium Al compositions, the n-AlGaN layer and QBs affect the LED performance in more complicated way. It is noteworthy that the Al composition of n-AlGaN layer also plays an important role in influencing the LED performance. The results of present study show that optimal output performance of DUV LEDs can be achieved by fine-tuning the three structural parameters. Detailed simulation results for the systematic analyses of DUV LED output performance will be presented in the Conference. Moreover, characterization for each LED structure and physical mechanisms relevant to the physical properties will be provided as well.

\section{References}

[1] V. Fiorentini, F. Bernardini, and O. Ambacher, "Evidence for nonlinear macroscopic polarization in III-V nitride alloy heterostructures," Appl. Phys. Lett., vol. 80, no. 7, pp. 1204-1206, 2002.

[2] D. A. B. Miller, D. S. Chemla, and T. C. Damen, "Band-edge electroabsorption in quantum well structure: The quantumconfined Stark effect," Phys. Rev. Lett., vol. 53, no. 22, pp. 2173-2176, 1984.

[3] Y.-K. Kuo, J.-Y. Chang, H.-T. Chang, F.-M. Chen, Y.-H. Shih, and B.-T. Liou, "Polarization effect in AlGaN-based deep-ultraviolet light-emitting diodes," IEEE J. Quantum Electron., vol. 53, no. 1, pp. 3300106-1-3300106-6, 2017. 